\title{
Dispersion of Interstellar Polarization
}

\author{
THOMAS GeHRELS \\ University of Arizona \\ Tucson, Arizona
}

THE WAVELENGTH DEPENDENCE OF POLARIZATION as observed in 132 stars, for which the Henry Draper numbers are given, is shown in figure 1. Details of some of these observations are presented in reterence 1 .

Ine equipment is now being used with the new 154-cm Catalina reflector of the Lunar and Planetary Laboratory at the University of Arizona. The instrumental polarizations are nearly zero. The data processing and observing techniques have been further improved; the precision is mainly determined by statistics such that the internal probable error in the percentage polarization is \pm 0.03 percent $( \pm 0.0006$ magnitude) for a half-hour observation per filter on objects brighter than about 7 magnitudes. The wavelength $\lambda$ ranges from 0.33 to $0.95 \mu$, covered by seven filters of bandwidth of about $0.05 \mu$. The wavelength range is being extended to $1.2,1.6$, and $2.2 \mu$, and, with high-altitude ballooning, to 0.28 and $0.22 \mu$.

The calculations for perfectly alined infinite cylinders in chapter 15 of reference 2 are fitted to these observations. The fits represent a first reconnaissance for which size distributions are ignored and the refractive index of dirty ice is adopted; it is then found that the particle diameters range between 0.2 and $0.4 \mu$. One or more interstellar clouds having such a narrow distribution in the refractive indices and particle sizes may give a curve similar to the one obtained for HD 2905 (fig. 1).

A flatter curve, such as that for HD 18326 in figure 1, may be caused by light passing through various clouds that also contain smaller particles, thereby raising the amount of polarization at the larger values of $\lambda^{-1}$. An upturn in the ultraviolet appears in a few cases (see, for example, the curve for HD 218342 in fig. 1); this upturn may be interpreted as evidence for additional clouds with particle diameters of about $0.17 \mu$.

An upturn in the infrared is similarly explained as showing evidence of larger particles. The strongest case is that of HD 37202 . It should be noted that HD 37041 in the Orion nebula is spectacularly different from the other stars.

$271-9920-67-5$ 

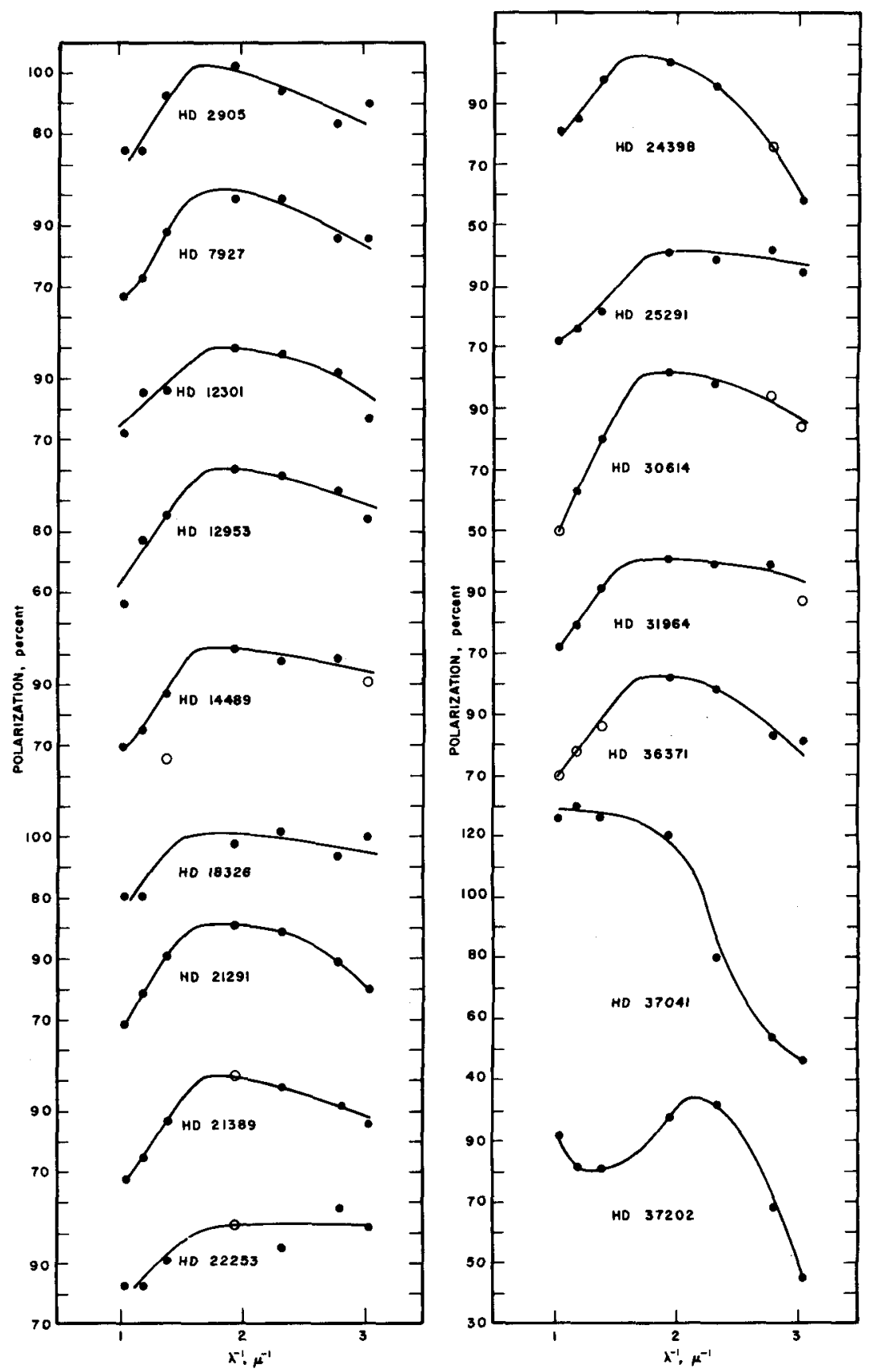

Figure 1. - Percentage polarization of selected stars, plotted after normalization to 100.0 for the straight average observed with filters at $\lambda^{-1}=1.95 \mu$ and $\lambda^{-1}=2.33 \mu$. 

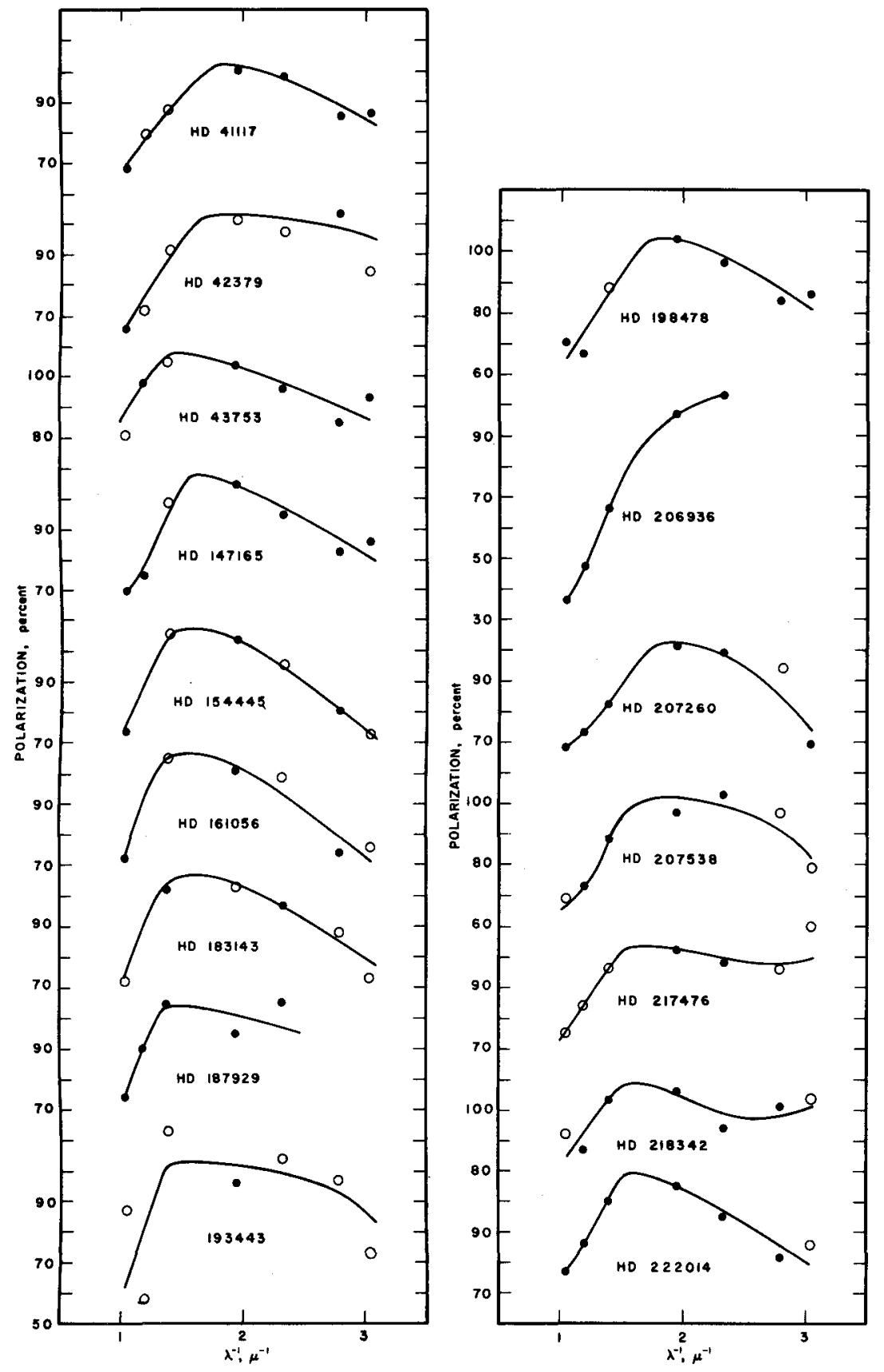

Figure 1. - Concluded. 
Also unusual is the curve for HD 206936 which is $\mu$ Cephei; the polarization is intrinsic, that is, caused within or close to the star itself. The visual polarization of $\mu$ Cep varies, with time, between 0.3 and 2.3 percent.

Polarimetry is a sensitive tool for the study of grain parameters. For various galactic regions, the differences between polarization curves are much more pronounced than the differences between photometric results.

The position angles also show wavelength dependence. Of the 35 stars observed to date, 14 show the effect appreciably; these stars are listed with their galactic longitudes in table I. For each star, the difference of the position angle (at each filter) from the mean position angle (of all seven filters) is listed in the table. A solution of nickel sulfate was used in the filter listed in the last column in order to eliminate red leakage.

The rotation of the position angle with wavelength is very puzzling. For the 14 stars selected for table I, the position angles increase with decreasing wavelength until the point perpendicular to the local spiral arm (longitude $=144^{\circ}$ ) is reached; and beyond that point the position angles decrease with decreasing wavelength. This effect is reminiscent

TABle I. - Difference Between Position Angle at Seven Filters and Mean Position Angle for 14 Stars

\begin{tabular}{|c|c|c|c|c|c|c|c|c|}
\hline \multirow[b]{2}{*}{ HD number } & \multirow[b]{2}{*}{$\begin{array}{c}\text { Galactic } \\
\text { longitude, } \\
\text { deg }\end{array}$} & \multicolumn{7}{|c|}{ Angular difference in degrees for- } \\
\hline & & $\begin{array}{c}\text { Infrared } \\
\text { filter } \\
(\lambda=0.95 \mu\end{array}$ & $\begin{array}{l}\text { Red } \\
\text { filter } \\
=0.84 \mu)\end{array}$ & $\begin{array}{c}\text { Orange } \\
\text { filter } \\
\lambda=0.71 \mu\end{array}$ & $\begin{array}{c}\text { Green } \\
\text { filter } \\
(\lambda=0.53 \mu)\end{array}$ & $\begin{array}{l}\text { Blue } \\
\text { filter } \\
=0.43\end{array}$ & $\left.\mid \begin{array}{c}\text { Ultraviolet } \\
\text { filter } \\
(\lambda=0.36 \mu\end{array}\right)$ & $\begin{array}{c}\text { Filter using } \\
\text { nickel sul- } \\
\text { fate solution } \\
(\lambda=0.33 \mu)\end{array}$ \\
\hline 147165 & 351 & $\mathrm{a}-2$ & $a-8$ & -2 & ${ }^{\mathrm{a}} 0$ & +1 & $a+6$ & +2 \\
\hline 207538 & 102 & -8 & -2 & a 0 & +2 & +2 & -1 & +6 \\
\hline 2905 & 121 & -5 & -3 & $a-4$ & -1 & +1 & +2 & +8 \\
\hline 7927 & 127 & -3 & -2 & -2 & 0 & $a+2$ & +1 & +5 \\
\hline 12301 & 131 & -3 & -2 & -2 & +1 & +1 & +2 & +3 \\
\hline 12953 & 133 & -6 & -2 & -2 & +1 & +2 & +3 & +4 \\
\hline 14489 & 136 & $a-6$ & -2 & -4 & 0 & +1 & $a+1$ & +7 \\
\hline 21389 & 142 & -2 & -1 & -1 & 0 & +1 & +1 & +2 \\
\hline 30614 & 144 & -3 & -1 & 0 & +1 & +2 & $a+1$ & +1 \\
\hline 22253 & 145 & +6 & +7 & +6 & 0 & o & -9 & -10 \\
\hline 24431 & 150 & +7 & +3 & +2 & -5 & +1 & -1 & -8 \\
\hline 36371 & 176 & $a+4$ & $a+2$ & +4 & $a+2$ & $a+1$ & -3 & -5 \\
\hline 37202 & 186 & $a+4$ & +4 & +7 & -1 & +1 & -4 & -9 \\
\hline 41117 & 190 & +4 & $a+2$ & $a+4$ & -1 & -3 & $a-1$ & $a-3$ \\
\hline
\end{tabular}

a Value obtained with poorer precision. 
of a Faraday rotation, but Faraday rotation should be negligible at optical wavelengths. As yet no explanation for this effect is known.

\section{REFERENCES}

1. Coyne, G. V.; and Gehrels, T.: Wavelength Dependence of Polarization. VIII. Interstellar Polarization. Astron. J., vol. 71, 1966, p. 355.

2. VAN De Hulst, H. C.: Light Scattering by Small Particles. John Wiley \& Sons, Inc., 1957.

\section{DISCUSSION}

Greenberg: Apparently the wavelength dependence of polarization becomes less and less peaked as we view further away from the perpendicular to the magnetic field. This observation seems to fit in somewhat with the remarks that have been made earlier so that, for example, the ratio of the polarization in the yellow to that in the blue would tend to be less when the line of sight is not quite perpendicular to the magnetic field than it would be if the line of sight were perpendicular to the magnetic field (high polarization).

I would like to comment on the terminology "dirty ice." Many things are used to represent dirty ice, and we should have some idea of where the imaginary part of the index of refraction comes from. In the visible we have made some calculations to see if an imaginary index of 0.05 is reasonable. The only way to get as much as 0.05 imaginary part in the visible index of refraction is to take all the iron in its usual cosmic abundance and put it in clumps large enough so that it acts like little pieces of metal.

All the other materials that are used for dielectrics are all very clear in the visible. In order for something to be called dirty, it should have an imaginary part of the order of 0.01 to 0.05 in the visible. In other words, anything having an imaginary part as large as 0.2 is metallic or is at least characteristic of what one may find in the neighborhood of a resonance or absorption line. Did you use 0.2 for the imaginary part?

Gehrels: Yes, we tried imaginary parts as large as that. In the paper on the reflection nebulae I conclude that acceptable model particles would be graphite particles covered with a coated shell. The other particle model that would fit the observations of NGC 7023 has a large imaginary part to the refractive index; in view of what you now say, then, the only remaining possibility is that of graphite particles with the ice coating.

Greenberg: Dirty ice is relatively clear in the visible. Even if it had an imaginary part between 0.01 to 0.05 and would thus look rather muddy, it would still be fairly clear as far as Mie calculations go.

Gehrels: Would you agree with the conclusion that the coated graphite particles give a good fit in general to the interstellar polarization? 
Greenberg: No.

Wickramasinghe: In order to get an imaginary part of 0.2 , a great deal of solid iron would have to be embedded in the ice.

Greenberg: Yes, you would need a lot of very small particles, all having common characteristics. These calculations which I made several years ago showed that embedding a number of iron atoms in the grains that is proportional to their cosmic abundance gave rise to a negligible contribution to the absorptivity even when all the iron absorption lines are included. In other words, a thick chunk of ice, even bigger than an ice cube, with a lot of iron atoms embedded. in it would still be transparent.

Spitzer: Is not $\theta^{2}$ Orionis (HD 37041) the star which has long been known for the unusual variation of selective extinction with wavelength?

Gehrels: Yes.

Spitzer: So everything makes sense, and the unusual wavelength dependence of polarization in this star is consistent with the presence of large particles, already indicated by the wavelength dependence of the interstellar reddening curve.

Field: What about $\lambda 4430$ ? If the particles really are $1 \mu$ in diameter, the absorption for a wavelength of $4430 \AA$ might be expected to be very anomalous; either it might be very weak or it might possibly even show up as an emission rather than absorption. What do the observations tell us?

Walker: The star HD 37041, if I remember correctly, shows a weak absorption for this wavelength.

Gehrels: Is there a correlation or connection between the $4430 \AA$ wavelength and the upturn in the interstellar reddening curve?

Nandy: We have found no correlation with the line intensity of $4430 \AA$ and the dispersion at that wavelength. If that upturn depended on $\lambda 4430$ we would expect the dispersion in extinction at that wavelength to correlate with the intensity of $\lambda 4430$.

Wickramasinghe: With regard to the isotropic conductivity assumption that you made, I think that, for a local region like the one you examined, all the graphite axes would almost surely be randomly oriented, and then I think that this assumption is as good as any other.

Nandy: Does the wavelength dependence of polarization depend on the galactic longitude, or is it just different for different groups of stars?

Gehrels: It is different for different stars. The curves for these different stars, including the relative errors, have been published in a series of papers in the Astronomical Journal.

Nandy: Is there any correlation between polarization and the wavelength of $4430 \AA$ ?

Gehrels: I do not know. We have not looked at that. 
Spitzer: We have heard about the great differences in the wavelength dependence of extinction and about the differences in the wavelength dependence of the polarization. Is there any clear-cut correlation between these differences? Have enough data been obtained?

Gehrels: We have made only a preliminary analysis and we see no correlation yet. Actually, the data do not overlap enough to make a study feasible. In Orion, however, there is at least an indication of larger particles from both the photonetry and from the polarization.

Spitzer: When you say Orion, are you referring primarily to the trapezium stars?

Gehrels: Yes.

Spitzer: As has already been emphasized, this is the region whose unusual properties have been known for some time. In this particular region there is a clear indication that these relatively large $1-\mu$ particles are present. For the rest of the sky are there still some uncertainties as to the possible presence of these larger particles?

Gehrels: Yes. Not enough stars have been observed yet.

Behr: Do you find the same color dependence in Orion as Dr. Appenzeller does?

Gehrels: I have not made a comparison with Dr. Appenzeller's observations as yet.

Greenberg: Dr. Gehrels refers to $1 \cdot \mu$ size particles; I assume that these particles are dielectric or dirty-ice particles. In other words, the wavelength dependence of polarization for HD 37202 implies that there are two distinct groups of particle sizes: one centered at about $0.4 \mu$ and the other, at about $1 \mu$. Why is there a gap between the two sizes?

Greenberg: There is doubt as to whether or not a discontinuity in the size distribution exists. If one used a model for grain growth and assumed that a particular cloud had existed without collisions for a longer time than usual, one would find considerably larger grains in this cloud than ordinarily would be found. The assumption of simultaneously smaller than average and larger than average particles as an explanation of the wavelength dependence of polarization due to a single cloud would be difficult to justify. However. in two separate clouds, one might reasonably expect two different size distributions. The net result for viewing through these clouds would then be equivalent to that of viewing through a single cloud.

Donn: A possible explanation for the rather sharp variation in size could be that in growing plates, the plate particle sizes are much more sensitive to possible density fluctuations in the medium than are, for example, spherical particles.

Walker: Are these particles large enough to explain the $\mu$ Cephei results and the neutral absorption suggested from the galaxy counts, or would larger particles be required to cause these phenomena? 
Greenberg: The polarization maximum at $\lambda^{-1} \approx 0.2 \mu^{-1}$ implies a dielectric grain whose size is about 10 times the normal size limit. This conclusion is also valid for relative sizes of metallic type grains.

Walker: So we have three distinct types of clouds of particles at the moment?

Greenberg: If the upturn in the infrared extinction is real, then this is possible. 